\title{
Evolutionary suicide and evolution of dispersal in structured metapopulations
}

Mats Gyllenberg (matsgyl@utu.fi)

Kalle Parvinen (kalparvi@utu.fi)

Ulf Dieckmann (dieckman@iiasa.ac.at)

\section{Approved by}

Arne Jernelöv (jernelov@iiasa.ac.at)

Acting Director, IIASA

November 2000 


\section{IIASA STUDIES IN ADAPTIVE DYNAMICS No. 48}

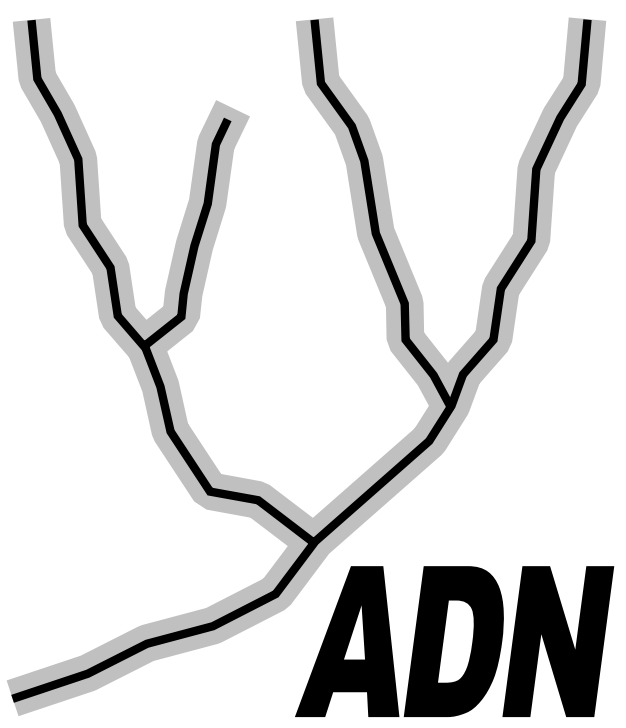

The Adaptive Dynamics Network at IIASA fosters the development of new mathematical and conceptual techniques for understanding the evolution of complex adaptive systems.

Focusing on these long-term implications of adaptive processes in systems of limited growth, the Adaptive Dynamics Network brings together scientists and institutions from around the world with IIASA acting as the central node.

Scientific progress within the network is reported in the IIASA Studies in Adaptive Dynamics series.

\section{THE ADAPTIVE DYNAMICS NETWORK}

The pivotal role of evolutionary theory in life sciences derives from its capability to provide causal explanations for phenomena that are highly improbable in the physicochemical sense. Yet, until recently, many facts in biology could not be accounted for in the light of evolution. Just as physicists for a long time ignored the presence of chaos, these phenomena were basically not perceived by biologists.

Two examples illustrate this assertion. Although Darwin's publication of "The Origin of Species" sparked off the whole evolutionary revolution, oddly enough, the population genetic framework underlying the modern synthesis holds no clues to speciation events. A second illustration is the more recently appreciated issue of jump increases in biological complexity that result from the aggregation of individuals into mutualistic wholes.

These and many more problems possess a common source: the interactions of individuals are bound to change the environments these individuals live in. By closing the feedback loop in the evolutionary explanation, a new mathematical theory of the evolution of complex adaptive systems arises. It is this general theoretical option that lies at the core of the emerging field of adaptive dynamics. In consequence a major promise of adaptive dynamics studies is to elucidate the long-term effects of the interactions between ecological and evolutionary processes.

A commitment to interfacing the theory with empirical applications is necessary both for validation and for management problems. For example, empirical evidence indicates that to control pests and diseases or to achieve sustainable harvesting of renewable resources evolutionary deliberation is already crucial on the time scale of two decades.

The Adaptive Dynamics Network has as its primary objective the development of mathematical tools for the analysis of adaptive systems inside and outside the biological realm. 


\section{IIASA STUDIES IN ADAPTIVE DYNAMICS}

No. 1 Metz JAJ, Geritz SAH, Meszéna G, Jacobs FJA, van Heerwaarden JS:

Adaptive Dynamics: A Geometrical Study of the Consequences of Nearly Faithful Reproduction.

IIASA Working Paper WP-95-099.

In: van Strien SJ, Verduyn Lunel SM (eds.): Stochastic and Spatial Structures of Dynamical Systems, Proceedings of the Royal Dutch Academy of Science (KNAW Verhandelingen), North Holland, Amsterdam, pp. 183-231 (1996).

No. 2 Dieckmann U, Law R:

The Dynamical Theory of Coevolution: A Derivation from Stochastic

Ecological Processes.

IIASA Working Paper WP-96-001.

Journal of Mathematical Biology (1996) 34, 579-612.

No. 3 Dieckmann U, Marrow P, Law R:

Evolutionary Cycling of Predator-Prey Interactions: Population Dynamics and the Red Queen.

Journal of Theoretical Biology (1995) 176, 91-102.

No. 4 Marrow P, Dieckmann U, Law R:

Evolutionary Dynamics of Predator-Prey Systems: An Ecological

Perspective.

IIASA Working Paper WP-96-002.

Journal of Mathematical Biology (1996) 34, 556-578.

No. 5 Law R, Marrow P, Dieckmann U:

On Evolution under Asymmetric Competition.

IIASA Working Paper WP-96-003.

Evolutionary Ecology (1997) 11, 485-501.

No. 6 Metz JAJ, Mylius SD, Diekmann O:

When Does Evolution Optimise? On the Relation between Types of Density Dependence and Evolutionarily Stable Life History Parameters.

IIASA Working Paper WP-96-004.

No. 7 Ferrière R, Gatto $\mathrm{M}$ :

Lyapunov Exponents and the Mathematics of Invasion in Oscillatory or Chaotic Populations.

Theoretical Population Biology (1995) 48, 126-171.

No. 8 Ferrière R, Fox GA:

Chaos and Evolution.

Trends in Ecology and Evolution (1995) 10, 480-485.

No. 9 Ferrière R, Michod RE:

The Evolution of Cooperation in Spatially Heterogeneous Populations.

IIASA Working Paper WP-96-029.

American Naturalist (1996) 147, 692-717. 
No. 10 Van Dooren TJM, Metz JAJ:

Delayed Maturation in Temporally Structured Populations with Non-Equilibrium Dynamics.

IIASA Working Paper WP-96-070.

Journal of Evolutionary Biology (1998) 11, 41-62.

No. 11 Geritz SAH, Metz JAJ, Kisdi É, Meszéna G:

The Dynamics of Adaptation and Evolutionary Branching.

IIASA Working Paper WP-96-077.

Physical Review Letters (1997) 78, 2024-2027.

No. 12 Geritz SAH, Kisdi É, Meszéna G, Metz JAJ:

Evolutionarily Singular Strategies and the Adaptive Growth and Branching of the Evolutionary Tree.

IIASA Working Paper WP-96-114.

Evolutionary Ecology (1998) 12, 35-57.

No. 13 Heino M, Metz JAJ, Kaitala V:

Evolution of Mixed Maturation Strategies in Semelparous Life-Histories: the Crucial Role of Dimensionality of Feedback Environment.

IIASA Working Paper WP-96-126.

Philosophical Transactions of the Royal Society of London Series B (1997) 352, 16471655.

No. 14 Dieckmann U:

Can Adaptive Dynamics Invade?

IIASA Working Paper WP-96-152.

Trends in Ecology and Evolution (1997) 12, 128-131.

No. 15 Meszéna G, Czibula I, Geritz SAH:

Adaptive Dynamics in a Two-Patch Environment: a Simple Model for

Allopatric and Parapatric Speciation.

IIASA Interim Report IR-97-001.

Journal of Biological Systems (1997) 5, 265-284.

No. 16 Heino M, Metz JAJ, Kaitala V:

The Enigma of Frequency-Dependent Selection.

IIASA Interim Report IR-97-061.

Trends in Ecology and Evolution (1998) 13, 367-370.

No. 17 Heino M:

Management of Evolving Fish Stocks.

IIASA Interim Report IR-97-062.

Canadian Journal of Fisheries and Aquatic Sciences (1998) 55, 1971-1982.

No. 18 Heino M:

Evolution of Mixed Reproductive Strategies in Simple Life-History Models.

IIASA Interim Report IR-97-063.

No. 19 Geritz SAH, van der Meijden E, Metz JAJ:

Evolutionary Dynamics of Seed Size and Seedling Competitive Ability.

IIASA Interim Report IR-97-071.

Theoretical Population Biology (1999) 55, 324-343. 
No. 20 Galis F, Metz JAJ:

Why are there so many Cichlid Species? On the Interplay of Speciation and Adaptive Radiation.

IIASA Interim Report IR-97-072.

Trends in Ecology and Evolution (1998) 13, 1-2.

No. 21 Boerlijst MC, Nowak MA, Sigmund K:

Equal Pay for all Prisoners. / The Logic of Contrition.

IIASA Interim Report IR-97-073.

AMS Monthly (1997) 104, 303-307.

Journal of Theoretical Biology (1997) 185, 281-294.

No. 22 Law R, Dieckmann U:

Symbiosis without Mutualism and the Merger of Lineages in Evolution.

IIASA Interim Report IR-97-074.

Proceedings of the Royal Society of London Series B (1998) 265, 1245-1253.

No. 23 Klinkhamer PGL, de Jong TJ, Metz JAJ:

Sex and Size in Cosexual Plants.

IIASA Interim Report IR-97-078.

Trends in Ecology and Evolution (1997) 12, 260-265.

No. 24 Fontana W, Schuster P:

Shaping Space: The Possible and the Attainable in RNA Genotype-Phenotype Mapping.

IIASA Interim Report IR-98-004.

Journal of Theoretical Biology (1998) 194, 491-515.

No. 25 Kisdi É, Geritz SAH:

Adaptive Dynamics in Allele Space: Evolution of Genetic Polymorphism by Small Mutations in a Heterogeneous Environment.

IIASA Interim Report IR-98-038.

Evolution (1999) 53, 993-1008.

No. 26 Fontana W, Schuster P:

Continuity in Evolution: On the Nature of Transitions.

IIASA Interim Report IR-98-039.

Science (1998) 280, 1451-1455.

No. 27 Nowak MA, Sigmund K:

Evolution of Indirect Reciprocity by Image Scoring. / The Dynamics of Indirect Reciprocity.

IIASA Interim Report IR-98-040.

Nature (1998) 393, 573-577.

Journal of Theoretical Biology (1998) 194, 561-574.

No. 28 Kisdi É:

Evolutionary Branching Under Asymmetric Competition.

IIASA Interim Report IR-98-045.

Journal of Theoretical Biology (1999) 197, 149-162.

No. 29 Berger U:

Best Response Adaptation for Role Games.

IIASA Interim Report IR-98-086. 
No. 30 Van Dooren TJM:

The Evolutionary Ecology of Dominance-Recessivity

IIASA Interim Report IR-98-096.

Journal of Theoretical Biology (1999) 198, 519-532.

No. 31 Dieckmann U, O'Hara B, Weisser W:

The Evolutionary Ecology of Dispersal.

IIASA Interim Report IR-98-108.

Trends in Ecology and Evolution (1999) 14, 88-90.

No. 32 Sigmund K:

Complex Adaptive Systems and the Evolution of Reciprocation.

IIASA Interim Report IR-98-100.

Ecosystems (1998) 1, 444-448.

No. 33 Posch M, Pichler A, Sigmund K:

The Efficiency of Adapting Aspiration Levels.

IIASA Interim Report IR-98-103.

Proceedings of the Royal Society of London Series B (1999) 266, 1427-1435.

No. 34 Mathias A, Kisdi É:

Evolutionary Branching and Coexistence of Germination Strategies.

IIASA Interim Report IR-99-014.

No. 35 Dieckmann U, Doebeli M:

On the Origin of Species by Sympatric Speciation.

IIASA Interim Report IR-99-013.

Nature (1999) 400, 354-357.

No. 36 Metz JAJ, Gyllenberg M:

How Should We Define Fitness in Structured Metapopulation Models? Including an Application to the Calculation of Evolutionarily Stable Dispersal Strategies.

IIASA Interim Report IR-99-019.

Research Report A39 (1999), University of Turku, Institute of Applied Mathematics, Turku, Finland.

No. 37 Gyllenberg M, Metz JAJ:

On Fitness in Structured Metapopulations.

IIASA Interim Report IR-99-037.

Research Report A38 (1999), University of Turku, Institute of Applied Mathematics, Turku, Finland.

No. 38 Meszéna G, Metz JAJ:

Species Diversity and Population Regulation: The Importance of Environmental Feedback Dimensionality.

IIASA Interim Report IR-99-045.

No. 39 Kisdi É, Geritz SAH:

Evolutionary Branching and Sympatric Speciation in Diploid Populations.

IIASA Interim Report IR-99-048.

No. 40 Ylikarjula J, Heino M, Dieckmann U:

Ecology and Adaptation of Stunted Growth in Fish.

IIASA Interim Report IR-99-050. 
No. 41 Nowak MA, Sigmund K:

Games on Grids.

IIASA Interim Report IR-99-038.

In: Dieckmann U, Law R, Metz JAJ (eds.): The Geometry of Ecological Interactions: Simplifying Spatial Complexity, Cambridge University Press, Cambridge, UK, pp. 135$150(2000)$.

No. 42 Ferrière R, Michod RE:

Wave Patterns in Spatial Games and the Evolution of Cooperation.

IIASA Interim Report IR-99-041.

In: Dieckmann U, Law R, Metz JAJ (eds.): The Geometry of Ecological Interactions: Simplifying Spatial Complexity, Cambridge University Press, Cambridge, UK, pp. 318$332(2000)$.

No. 43 Kisdi É, Jacobs FJA, Geritz SAH:

Red Queen Evolution by Cycles of Evolutionary Branching and Extinction.

IIASA Interim Report IR-00-030.

No. 44 Meszéna G, Kisdi É, Dieckmann U, Geritz SAH, Metz JAJ:

Evolutionary Optimisation Models and Matrix Games in the Unified Perspective of Adaptive Dynamics.

IIASA Interim Report IR-00-039.

No. 45 Parvinen K, Dieckmann U, Gyllenberg M, Metz JAJ:

Evolution of Dispersal in Metapopulations with Local Density Dependence and Demographic Stochasticity.

IIASA Interim Report IR-00-035.

No. 46 Doebeli M, Dieckmann U:

Evolutionary Branching and Sympatric Speciation Caused by Different Types of Ecological Interactions.

IIASA Interim Report IR-00-040.

No. 47 Heino M, Hanski I:

Evolution of Migration Rate in a Spatially Realistic Metapopulation Model.

IIASA Interim Report IR-00-044.

No. 48 Gyllenberg M, Parvinen K, Dieckmann U:

Evolutionary Suicide and Evolution of Dispersal in Structured Metapopulations.

IIASA Interim Report IR-00-056.

Issues of the IIASA Studies in Adaptive Dynamics series can be obtained free of charge. Please contact:

Adaptive Dynamics Network

International Institute for Applied Systems Analysis

Schlossplatz 1

A-2361 Laxenburg

Austria

Telephone +432236 807, Telefax +43 2236 71313, E-Mail adn@iiasa.ac.at, Internet http://www.iiasa.ac.at/Research/ADN 


\section{Contents}

1 Introduction $\quad 1$

2 Model description $\quad 1$

2.1 Resident dynamics . . . . . . . . . . . . . . . . . . . . . 2

2.2 Resident equilibria . . . . . . . . . . . . . . . . 3

2.3 Basic reproduction ratio of the resident $\ldots \ldots \ldots \ldots$

2.4 Invasion fitness of the mutant . . . . . . . . . . . . . 5

3 Without catastrophes the strategy not to disperse is an ESS 5

4 Continuous transition to extinction $\quad 7$

4.1 Limit patch size distribution . . . . . . . . . . . . . . . . 7

4.2 Evolutionary repulsion of the extinction boundary $\ldots \ldots \ldots$. . . . 8

4.3 First example: Constant catastrophe rate . . . . . . . . . . . . . 8

5 Discontinuous transition to extinction 9

5.1 A discontinuous transition to extinction is necessary for evolutionary suicide 9

5.2 Second example: Size-dependent catastrophe rate . . . . . . . . . . . . 10

5.3 Third example: Allee effect . . . . . . . . . . . . . . . . . . . 11

5.4 A discontinuous transition to extinction is not sufficient for evolutionary suicide . . . . . . . . . . . . . . . . . . 12

6 Discussion 13

A Cumulative formulation of the model $\quad 16$ 


\begin{abstract}
In this article we study the evolution of dispersal in a structured metapopulation model. The metapopulation consists of a large (infinite) number of local populations living in patches of habitable environment. Dispersal between patches is modelled by a disperser pool and individuals in transit between patches are exposed to a risk of mortality. Occasionally, local catastrophes eradicate a local population: all individuals in the affected patch die, yet the patch remains habitable. The rate at which such disasters occur can depend on the local population size of a patch. We prove that, in the absence of catastrophes, the strategy not to migrate is evolutionarily stable. It is also convergence stable unless there is no mortality during dispersal. Under a given set of environmental conditions, a metapopulation may be viable and yet selection may favor dispersal rates that drive the metapopulation to extinction. This phenomenon is known as evolutionary suicide. We show that in our model evolutionary suicide can occur for certain types of size-dependent catastrophes. Evolutionary suicide can also happen for constant catastrophe rates, if local growth within patches shows an Allee effect. We study the evolutionary bifurcation towards evolutionary suicide and show that a discontinuous transition to extinction is a necessary condition for evolutionary suicide to occur. In other words, if population size smoothly approaches zero at a boundary of viability in parameter space, this boundary is evolutionarily repelling and no suicide can occur.
\end{abstract}




\title{
About the Authors
}

\author{
Mats Gyllenberg \\ Department of Mathematics \\ FIN-20014 University of Turku, Finland \\ and \\ Turku Centre for Computer Science \\ FIN-20014 University of Turku, Finland \\ Kalle Parvinen \\ Department of Mathematics \\ FIN-20014 University of Turku, Finland \\ and \\ Adaptive Dynamics Network \\ International Institute for Applied Systems Analysis \\ A-2361 Laxenburg \\ Austria \\ Ulf Dieckmann \\ Adaptive Dynamics Network \\ International Institute for Applied Systems Analysis \\ A-2361 Laxenburg \\ Austria
}

\section{Acknowledgements}

The authors wish to thank Régis Ferrière for sharing his ideas about evolutionary suicide. Much of the original motivation for this paper derives from discussions with him and talks given by him. We have also greatly benefited from collaboration with Hans Metz over the years. This work was carried out when Kalle Parvinen was participating in the Young Scientists Summer Program at IIASA, Laxenburg, Austria, and was partially funded by the Academy of Finland. 


\title{
Evolutionary suicide and evolution of dispersal in structured metapopulations
}

\author{
Mats Gyllenberg \\ Kalle Parvinen* \\ Ulf Dieckmann
}

\section{Introduction}

Most natural populations live in heterogeneous environments. A metapopulation consists of several local populations living in habitat patches. In the classical Levins $(1969,1970)$ metapopulation model, local population dynamics are neglected and habitat patches are assumed to be equal in all respects. In nature this is usually not the case, therefore structured metapopulation models (Gyllenberg et al. 1997; Gyllenberg and Hanski 1992, 1997; Hanski and Gyllenberg 1993, 1997) are more realistic and more interesting to study.

Dispersal between habitat patches is a key feature of metapopulation ecology. Recently, the evolution of dispersal has raised a lot of interest (Gandon 1999; Gandon and Michalakis 1999; Gyllenberg and Metz 1999; Heino and Hanski 2000; Metz and Gyllenberg 2001; Parvinen 1999; Parvinen et al. 2000; Ronce et al. 2000). We study the evolution of dispersal in a metapopulation evolving according to the model of Gyllenberg and Metz (1999) and Metz and Gyllenberg (2001).

The paper is structured as follows. In Section 2 we present the metapopulation model and derive expressions for steady states, the basic reproduction ratio and invasion fitness. In Section 3 we show that without catastrophes the strategy not to disperse is evolutionarily stable. It is also convergence stable (Christiansen 1991), i.e., it is an evolutionary attractor, unless there is no mortality during dispersal. A transition from a viable parameter region to a region predicting extinction can occur in several different ways, i.e., through different kinds of bifurcation. In Section 4 we study transitions to extinction where the equilibrium immigration rate, and thus the total metapopulation size, continuously goes to zero as the dispersal rate varies. We show that for such continuous transitions to extinction the boundaries of viability are evolutionarily repelling: therefore evolutionary suicide cannot occur. In Section 5 we study discontinuous transititions to extinction. We present two ecological scenarios that can give rise to evolutionary suicide. We prove and illustrate that a discontinuous transition to extinction is a necessary, but not a sufficient condition for evolutionary suicide.

\section{Model description}

We consider a metapopulation with a large number of identical habitat patches. This number is assumed to be large enough for the metapopulation dynamics to be well approximated by a metapopulation with infinitely many patches. Each patch can support a

\footnotetext{
${ }^{*}$ Author for correspondence
} 
local population. The metapopulation is structured by the size $x$ of local populations. The state of the metapopulation is thus the distribution $n$ of local populations (Gyllenberg et al. 1997). The size $x$ of local populations grows or decreases due to birth and death events according to local density regulation with a per capita growth rate $g(x)$. Patches are connected by dispersal: individuals leave their patch at a rate $k(x)$ and enter a disperser pool. $k(x)$ is the emigration rate or dispersal rate. The density of dispersers is denoted by $D$. Individuals in the disperser pool experience mortality at a per capita rate $\nu$ and they leave the pool and immigrate into a patch at a per capita rate $\alpha$. The quantity

$$
I=\alpha D
$$

is the immigration rate to each patch, which for simplicity we from now on call immigration rate. The quantity

$$
\pi=\frac{\alpha}{\alpha+\nu}
$$

is the probability that a disperser survives migration. Correspondingly, $\rho=1-\pi=$ $\nu /(\alpha+\nu)$ is the probability that a disperser dies during migration. We therefore refer to $\rho$ as the dispersal risk.

Local populations may go extinct as a result of catastrophes, which are assumed to occur at a size-specific rate $\mu(x)$. After a catastrophe, the patch is immediately recolonized by migrants from the disperser pool. We assume that the rates $g, k$, and $\mu$ are continuous functions of local population size.

In the following subsections, we first investigate the dynamics of a metapopulation of resident individuals with dispersal rate $k(x)$, and then analyze how the resident metapopulation responds to the arrival of mutants with a different dispersal rate $k_{\text {mut }}(x)$. All theorems apply for size-dependent dispersal strategies, but in the examples dispersal is assumed to be constant $k(x)=k$.

\section{$2.1 \quad$ Resident dynamics}

As our basic metapopulation model we use the one introduced by Gyllenberg and Metz 1999. The local population growth, including emigration and immigration, is given by

$$
\frac{d x}{d t}=f(x, I(t))
$$

with

$$
f(x, I)=g(x) x-k(x) x+I .
$$

At the metapopulation level, we study the density $n(t, x)$ of local population sizes $x$. The dynamics of this density can be described by the partial differential equation,

$$
\frac{\partial}{\partial t} n(t, x)+\frac{\partial}{\partial x}[f(x, I(t)) n(t, x)]=-\mu(x) n(t, x),
$$

which has to obey the following side condition at $x=0$ :

$$
I(t) n(t, 0)=\int_{0}^{\infty} \mu(x) n(t, x) d x .
$$

The dynamics of the immigration rate is given by

$$
\frac{d}{d t} I(t)=-(\alpha+\nu) I(t)+\alpha \int_{0}^{\infty} k(x) x n(t, x) d x .
$$


As a matter of fact, we consider the model (5)-(7) as a shorthand notation for a mathematically precise model in the so-called cumulative formulation (Diekmann et al. 1998,2001; Gyllenberg et al. 1997).

One of the reasons for this is that the metapopulation state corresponding to metapopulation extinction is the point mass concentrated at the origin and this cannot be represented by a density $n$. Another reason is that we shall consider the situation of no catastrophes $(\mu=0)$, in which case the boundary condition (6) becomes meaningless. And last but not least, it follows from the results by Diekmann et al. (2001) that the model in the cumulative formulation is well-posed. Diekmann et al. (2000) gave examples of innocent looking hyperbolic systems very similar to (5)-(7), that lack unique solutions. We refer to Gyllenberg and Metz (1999) for a complete cumulative model formulation. In the Appendix we derive expressions for the steady states in the cases $\mu=0$ and $I=0$, which require the cumulative formulation.

\subsection{Resident equilibria}

For a given constant value $I>0$ of the input, we infer from (5)-(6) that the unique distribution of local population sizes satisfying $\frac{\partial}{\partial t} n(t, x)=0$ is

$$
n(x, I)= \begin{cases}\frac{1}{l(I)} \frac{\Pi(x, I)}{f(x, I)} & \text { if } x<\tilde{x}(I) \\ 0 & \text { otherwise }\end{cases}
$$

where

$$
\tilde{x}(I)=\inf \{x>0 \mid f(x, I)=0\}
$$

is the supremum of attainable sizes. Two other functions are used in (8). First,

$$
\Pi(x, I)=\exp \left[-\int_{0}^{x} \frac{\mu(\xi)}{f(\xi, I)} d \xi\right]
$$

is the probability that a local population will survive at least to size $x$. Second,

$$
l(I)=\int_{0}^{\tilde{x}(I)} \frac{1}{f(x, I)} \Pi(x, I) d x
$$

is the expected lifetime of a local population. From (7) we see that the equilibrium immigration rate must satisfy the balance equation

$$
I=\pi \int_{0}^{\tilde{x}(I)} k(x) x n(x, I) d x=\pi \frac{E(I)}{l(I)}
$$

where

$$
E(I)=\int_{0}^{\tilde{x}(I)} \frac{k(x) x}{f(x, I)} \Pi(x, I) d x
$$

denotes the expected number of dispersers produced by a local population during its entire life. Equation (12) therefore has the natural interpretation that, at equilibrium, the immigration rate equals the emigration rate times the probability of surviving migration. We denote equilibrium inputs, that is, solutions of (12), by $I^{*}$. The equilibrium local population size distribution corresponding to $I^{*}$ is

$$
n^{*}(x)=n\left(x, I^{*}\right) .
$$


(a)

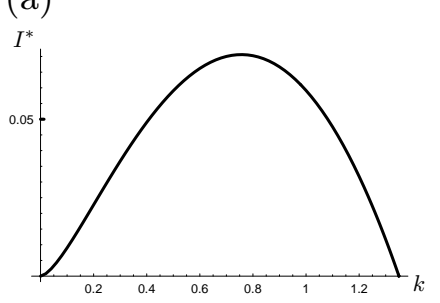

(b)

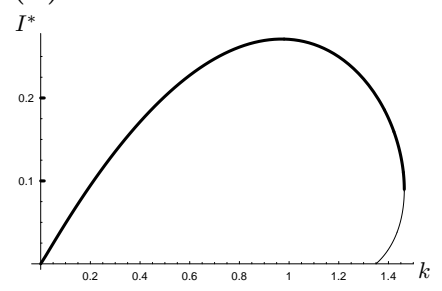

(c)

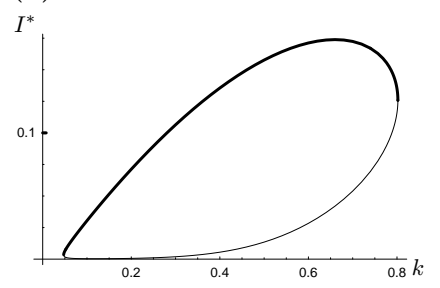

Figure 1: Dependence of the equilibrium immigration rate $I^{*}$ on the dispersal rate $k$ in different settings. (a) Constant catastrophes $\mu(x)=0.4$. (b) \& (c) Catastrophe rate $\mu$ decreases with local population size $x$ according to (36) with $\mu(0)=0.4$ in (b) and $\mu(0)=1.2$ in (c). Stable and unstable equilibria $I^{*}$ are shown as thick and thin curves, respectively. Local growth is logistic with $g(x)=1-x$. Parameters: $\alpha=0.5, \nu=0.4$.

\subsection{Basic reproduction ratio of the resident}

We define the basic reproduction ratio $R$ as the mean number of arriving offspring produced over the lifetime of a local population divided by the mean number of arrivals. When the input $I$ is constant, the mean number of arrivals equals $l(I) I$. Thus we obtain

$$
R^{\mathrm{res}}(I)=\pi \frac{E(I)}{l(I) I}
$$

Observe that the steady-state condition (12) reduces to $R^{\text {res }}(I)=1$, which means that every arriving disperser exactly replaces itself. For a more abstract way of defining $R^{\text {res }}(I)$ as the spectral radius of the next-generation operator we refer to Diekmann et al. (1998), Gyllenberg et al. (1997) and Gyllenberg and Metz (1999).

The number

$$
R_{0}^{\mathrm{res}}:=\lim _{I \rightarrow 0} R^{\mathrm{res}}(I)
$$

determines whether the resident population can spread in a virgin environment.

Theorem 1. The number $R_{0}^{\mathrm{res}}$ equals

$$
R_{0}^{\mathrm{res}}= \begin{cases}\pi \frac{k(0)}{\mu(0)-g(0)+k(0)} & \text { if } \mu(0)-g(0)+k(0)>0 \\ \infty & \text { otherwise }\end{cases}
$$

Proof. Consider the quantity

$$
\bar{E}(I)=\frac{E(I)}{I}=\int_{0}^{\tilde{x}(I)} \frac{k(x) x}{I f(x, I)} \Pi(x, I) d x .
$$

By a change of variables, $z=x / I$, we obtain

$$
\bar{E}(I)=\int_{0}^{\tilde{x}(I) / I} \frac{k(z I) z}{[g(z I)-k(z I)] z+1} \exp \left[-\int_{0}^{z} \frac{\mu(\eta I)}{[g(\eta I)-k(\eta I)] \eta+1} d \eta\right] d z .
$$

Let $I \rightarrow 0$ to get

$$
\begin{aligned}
\lim _{I \rightarrow 0} \bar{E}(I) & =\int_{0}^{\infty} \frac{k(0) z}{[g(0)-k(0)] z+1} \exp \left[-\int_{0}^{z} \frac{\mu(0)}{[g(0)-k(0)] \eta+1} d \eta\right] d z \\
& =\frac{k(0)}{\mu(0)[\mu(0)-g(0)+k(0)]} .
\end{aligned}
$$

The result (17) follows from this, from equation (15), and from the fact that $\lim _{I \rightarrow 0} l(I)=$ $1 / \mu(0)$. 
If $R_{0}^{\text {res }}>1$, the metapopulation is viable. The model can have multiple equilibria, as can be seen in Figs. 1b and 1c. This was already noted by Gyllenberg and Hanski (1992) for a very similar model with a different colonization rule. Therefore, $R_{0}^{\text {res }}<1$ does not necessarily imply extinction.

\subsection{Invasion fitness of the mutant}

When a mutant arrives in a patch where the resident population has size $x_{\text {res }}^{0}$, it feels the local population size $X_{I}\left(t, x_{\mathrm{res}}^{0}\right)$, where $X_{I}\left(t, x_{\mathrm{res}}^{0}\right)$ is the solution of $(3)$ with $x(0)=x_{\mathrm{res}}^{0}$. The expected number of dispersers per individual produced by a mutant population can be written in two integral forms. In the first one the integration variable is the age of a mutant colony, in the other one it is the resident population size;

$$
\begin{aligned}
E^{\mathrm{mut}}\left(x_{\mathrm{res}}^{0}, I\right)= & \int_{0}^{\infty} k_{\mathrm{mut}}\left(X_{I}\left(t, x_{\mathrm{res}}^{0}\right)\right) \exp \left[\int_{0}^{t} g\left(X_{I}\left(\tau, x_{\mathrm{res}}^{0}\right)\right)\right. \\
& \left.\quad-k_{\mathrm{mut}}\left(X_{I}\left(\tau, x_{\mathrm{res}}^{0}\right)\right)-\mu\left(X_{I}\left(\tau, x_{\mathrm{res}}^{0}\right)\right) d \tau\right] d t \\
= & \int_{x_{\mathrm{res}}^{0}}^{\tilde{x}(I)} \frac{k_{\mathrm{mut}}(x)}{f(x, I)} \exp \left[\int_{x_{\mathrm{res}}^{0}}^{x} \frac{g(\xi)-k_{\mathrm{mut}}(\xi)-\mu(\xi)}{f(\xi, I)} d \xi\right] d x .
\end{aligned}
$$

The fitness of the mutant is therefore given by

$$
\begin{aligned}
R^{\mathrm{mut}}\left(I^{*}\right) & =\pi \int_{0}^{\tilde{x}(I)} E^{\mathrm{mut}}\left(x_{\mathrm{res}}, I^{*}\right) n^{*}\left(x_{\mathrm{res}}\right) d x_{\mathrm{res}} \\
& =\frac{\pi}{l\left(I^{*}\right)} \int_{0}^{\infty} E^{\mathrm{mut}}\left(X_{I^{*}}\left(t, x_{\mathrm{res}}\right), I^{*}\right) \Pi\left(X_{I^{*}}\left(t, x_{\mathrm{res}}\right), I^{*}\right) d t .
\end{aligned}
$$

Based on this invasion fitness, which was derived by Gyllenberg and Metz (1999), framework of adaptive dynamics (Metz et al. 996a; Geritz et al. 1997,1998) to analyze the evolutionary dynamics of the metapopulation.

A strategy $k^{*}$ is an evolutionarily stable strategy (ESS) if it cannot be invaded by other strategies (Maynard Smith and Price 1973, Maynard Smith 1982). A strategy $k^{*}$ is convergence stable or an evolutionary attractor if the repeated invasion of nearby mutant strategies into nearby resident strategies will lead to the convergence of resident strategies towards $k^{*}$ (Christiansen 1991). A strategy that is both convergence stable and evolutionarily stable is a continuously stable strategy or CSS (Eshel 1983). For the distinction between evolutionary and convergence stability see also Taylor (1989). This terminology might not be ideal, but has nevertheless become established in the literature.

\section{Without catastrophes the strategy not to disperse is an ESS}

If there are no catastrophes, populations in all patches will grow towards the supremum size $\tilde{x}\left(I^{*}\right)$. It is therefore intuitively clear that the equilibrium local population size distribution will be $\delta_{\tilde{x}\left(I^{*}\right)}$, a fact that will be proved in Lemma 1. At a demographic equilibrium there is no advantage to dispersal; a dispersing individual will arrive at a patch that has the same conditions as the one it has left. In fact, we shall prove that the strategy not to disperse is evolutionarily stable in the absence of catastrophes. Furthermore, we shall prove that it is a continuously stable strategy (CSS) if, in the absence of catastrophes, there is positive dispersal risk: $\rho>0$. 
The equilibrium distribution $n^{*}$ of local population sizes found in (14) is a density, that is, an element of $L^{1}\left(\mathbb{R}_{+}\right)$. By the usual (Radon-Nikodym) embedding of $L^{1}$ into $M$ (the space of all Borel measures), densities can be regarded as absolutely continuous measures. In particular, it makes sense to speak about weak*-convergence of densities.

Lemma 1. Assume $\mu(x)=\mu \geqslant 0$ is independent of $x$. If $\mu=0$, then $n^{*}=\delta_{\tilde{x}\left(I^{*}\right)}$. Moreover, $n^{*}$ tends to $\delta_{\tilde{x}(I)}$ in the weak ${ }^{*}$-topology as $\mu \rightarrow 0$.

Proof. That $\mu=0$ implies $n^{*}=\delta_{\tilde{x}(I)}$ is proved in the Appendix.

When $\mu(x)=\mu \neq 0$ then $l(I)=1 / \mu$. Let $H(x)$ be continuously differentiable. Integrating by parts, we obtain

$$
\begin{aligned}
G(\mu, H) & =\int_{0}^{\tilde{x}(I)} H(x) n^{*}(x) d x=\int_{0}^{\tilde{x}(I)} H(x) \frac{\mu}{f(x, I)} \Pi(x, I) d x \\
& =H(0)+\int_{0}^{\tilde{x}(I)} H^{\prime}(x) \Pi(x, I) d x .
\end{aligned}
$$

The integral is bounded and $\Pi(x, I) \rightarrow 1$ for $x<\tilde{x}(I)$ when $\mu \rightarrow 0$. By Lebesque's dominated convergence theorem we have

$$
\lim _{\mu \rightarrow 0} G(\mu, H)=H(0)+\int_{0}^{\tilde{x}(I)} H^{\prime}(x) d x=H(\tilde{x}(I)) .
$$

Since continuously differentiable functions are dense in the set of continuous functions, the claim $\lim _{\mu \rightarrow 0} G(\mu, H)=H(\tilde{x}(I))$ holds also when $H$ is only continuous.

Using the weak*-convergence of the local population size distribution we can evaluate explicitly the fitness of rare mutants and conduct the evolutionary analysis in the absence of catastrophes.

Theorem 2. If $\mu=0$, the non-dispersing strategy $k(x)=0$ is evolutionarily stable; it is also an evolutionary attractor (convergence stable) if $\nu>0$.

Proof. According to Lemma 1, the fitness of the mutant is

$$
\begin{aligned}
R^{\mathrm{mut}}\left(I^{*}\right) & =\pi E^{\mathrm{mut}}\left(\tilde{x}\left(I^{*}\right), I^{*}\right) \\
& =\pi \int_{0}^{\infty} k_{\mathrm{mut}}\left(\tilde{x}\left(I^{*}\right)\right) \exp \left[\int_{0}^{t} g\left(\tilde{x}\left(I^{*}\right)\right)-k_{\mathrm{mut}}\left(\tilde{x}\left(I^{*}\right)\right) d \tau\right] d t \\
& = \begin{cases}\pi \frac{k_{\mathrm{mut}}\left(\tilde{x}\left(I^{*}\right)\right)}{k_{\mathrm{mut}}\left(\tilde{x}\left(I^{*}\right)\right)-g\left(\tilde{x}\left(I^{*}\right)\right)} & \text { if } k_{\mathrm{mut}}\left(x\left(\tilde{I} I^{*}\right)\right)-g\left(\tilde{x}\left(I^{*}\right)\right)>0 \\
\infty & \text { if } k_{\mathrm{mut}}\left(\tilde{x}\left(I^{*}\right)\right)-g\left(\tilde{x}\left(I^{*}\right)\right)<0 .\end{cases}
\end{aligned}
$$

As proved in the Appendix, $I^{*}=\pi k\left(\tilde{x}\left(I^{*}\right)\right) \tilde{x}\left(I^{*}\right)$. Therefore, from $f\left(\tilde{x}\left(I^{*}\right), I^{*}\right)=0$,

$$
g\left(\tilde{x}\left(I^{*}\right)\right)=k\left(\tilde{x}\left(I^{*}\right)\right)-\frac{I^{*}}{\tilde{x}\left(I^{*}\right)}=\rho k\left(\tilde{x}\left(I^{*}\right)\right) .
$$

Therefore, when $k_{\text {mut }}\left(\tilde{x}\left(I^{*}\right)\right)>\rho k\left(\tilde{x}\left(I^{*}\right)\right)$, we have

$$
R_{0}^{\mathrm{mut}}\left(I^{*}\right)=\frac{\alpha k_{\mathrm{mut}}\left(\tilde{x}\left(I^{*}\right)\right)}{\alpha k_{\mathrm{mut}}\left(\tilde{x}\left(I^{*}\right)\right)+\nu\left[k_{\mathrm{mut}}\left(\tilde{x}\left(I^{*}\right)\right)-k_{\mathrm{res}}\left(\tilde{x}\left(I^{*}\right)\right)\right]} .
$$

The case $\nu=0$ is neutral, since then $R_{0}^{\operatorname{mut}}\left(I^{*}\right)=1$. If $\nu>0$ we easily see

$$
R_{0}^{\mathrm{mut}}\left(I^{*}\right)\left\{\begin{array}{cl}
=1 & \text { if } k_{\mathrm{mut}}\left(\tilde{x}\left(I^{*}\right)\right)=k_{\mathrm{res}}\left(\tilde{x}\left(I^{*}\right)\right) \\
>1 & \text { if } k_{\mathrm{mut}}\left(\tilde{x}\left(I^{*}\right)\right)<k_{\mathrm{res}}\left(\tilde{x}\left(I^{*}\right)\right) \\
<1 & \text { if } k_{\mathrm{mut}}\left(\tilde{x}\left(I^{*}\right)\right)>k_{\mathrm{res}}\left(\tilde{x}\left(I^{*}\right)\right)
\end{array}\right.
$$

and $R_{0}^{\mathrm{mut}}\left(I^{*}\right)=\pi=\frac{\alpha}{\alpha+\nu}<1$ if $k_{\text {res }}\left(\tilde{x}\left(I^{*}\right)\right)=0$. Therefore, the strategy not to disperse is continuously stable. 
(a) Supercritical bifurcation

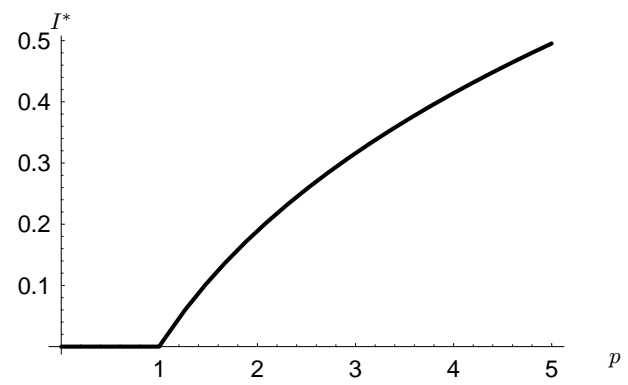

(b) Subcritical bifurcation

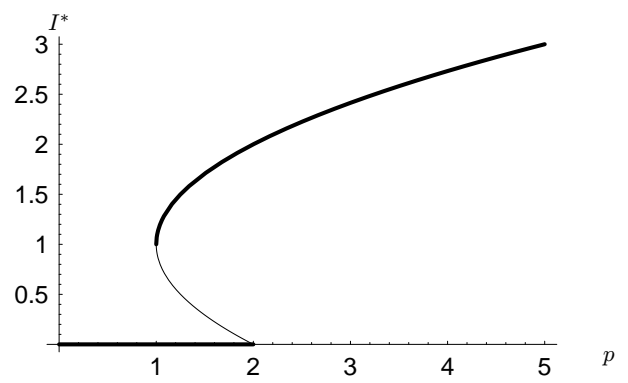

Figure 2: Typical examples of (a) continuous and (b) discontinuous transitions to extinction. The equilibrium immigration rate $I^{*}$ is plotted against some parameter $p$. Stable equilibria lie on the thick curve, unstable ones on the thin curve.

The evolutionary stability of zero dispersal in the absence of catastrophes has been studied before. Hastings (1983) found this in a continuous-time model with a finite number of patches. Parvinen (1999) investigated a discrete-time metapopulation model with a finite number of patches without catastrophes and proved that within the model the strategy not to migrate is a CSS, if the resident is at a fixed point equilibrium and there is mortality during dispersal.

\section{Continuous transition to extinction}

The metapopulation is not viable for all parameter values. The change from viability to extinction can happen through several different bifurcations. In this section we study transitions to extinction, for which the equilibrium immigration rate goes to zero continuously as the dispersal rate $k$ varies. A typical example is the situation in which the solution corresponding to metapopulation extinction $\left(I^{*}=0\right)$ loses its stability through a supercritical bifurcation (see Fig. 2a).

\subsection{Limit patch size distribution}

If the immigration rate $I$ becomes zero, patches will not be recolonized after a catastrophe has occurred. Accordingly, the corresponding equilibrium size distribution $n^{*}$ is the trivial one, $\delta_{0}$, with all patches being empty. Our next lemma shows that $n^{*}$ continuously depends on $I$ at $\delta_{0}$ with respect to the weak*-topology.

Lemma 2. $I=0$ implies $n^{*}=\delta_{0}$. Furthermore $n^{*} \rightarrow \delta_{0}$ in the weak ${ }^{*}$-topology as $I^{*} \rightarrow 0$.

Proof. That $I=0$ implies $n^{*}=\delta_{0}$ is proven in the Appendix.

Let $H(x)$ be a continuously differentiable function. By partial integration we get

$$
\int_{0}^{\tilde{x}(I)} H(x) \frac{\Pi(x, I)}{f(x, I)} d x=\frac{H(0)}{\mu(0)}+\int_{0}^{\tilde{x}(I)} \frac{d}{d x}\left[\frac{H(x)}{\mu(x)}\right] \Pi(x, I) d x .
$$

The last integral is bounded, therefore Lebesques dominated convergence theorem can be used. Since $\Pi(0, I)=1$ and $\lim _{I \rightarrow 0} \Pi(x, I)=0$ when $x>0$, the last integral tends to zero for $I \rightarrow 0$. The integral

$$
\int_{0}^{\tilde{x}(I)} H(x) \frac{\Pi(x, I)}{f(x, I)} d x \rightarrow \frac{H(0)}{\mu(0)}
$$


for $I \rightarrow 0$, if $H / \mu$ is bounded. Take $H(x)=1$ to obtain $\lim _{I \rightarrow 0} l(I)=1 / \mu(0)$. Therefore

$$
\int_{0}^{\tilde{x}(I)} H(x) n^{*}(x) d x=\int_{0}^{\tilde{x}(I)} H(x) \frac{\Pi(x, I)}{l(I) f(x, I)} d x \rightarrow H(0)
$$

for $I \rightarrow 0$. As in the proof of Lemma 1, the claim holds also when $H$ is only continuous, since continuously differentiable functions are dense in the set of continuous functions.

\subsection{Evolutionary repulsion of the extinction boundary}

When the resident population size is low, the mutant metapopulation will grow and produce dispersers as if it were in a virgin environment. Therefore the mutant's fitness equals $R_{0}^{\text {res }}$. This is proven in the following lemma.

\section{Lemma 3.}

$$
\lim _{I \rightarrow 0} R^{\mathrm{mut}}(I)=\left.R_{0}^{\mathrm{res}}\right|_{k_{\mathrm{res}}=k_{\mathrm{mut}}} .
$$

Proof. According to Lemma 2

$$
\lim _{I \rightarrow 0} R^{\mathrm{mut}}(I)=\pi \lim _{I \rightarrow 0} E^{\mathrm{mut}}(0, I)
$$

The result (32) follows from

$$
\begin{aligned}
E^{\mathrm{mut}}(0,0) & =\int_{0}^{\infty} k_{\mathrm{mut}}(0) \exp \left[\int_{0}^{t}\left[g(0)-k_{\mathrm{mut}}(0)-\mu(0)\right] d \tau\right] d t \\
& = \begin{cases}\frac{k_{\mathrm{mut}}(0)}{k_{\mathrm{mut}}(0)+\mu(0)-g(0)}, & \text { if } k_{\mathrm{mut}}(0)+\mu(0)-g(0)>0 \\
\infty & \text { otherwise }\end{cases}
\end{aligned}
$$

For those readers interested in adaptive dynamics theory we mention that Lemma 3 is very closely related to the assumption S3 in section 6.3.3 of Metz et al. (996a).

If the value of an evolving parameter $p$ is at the boundary $\partial V$ of the viability region $V$, then mutants that are viable in the absence of the resident can invade. Mutants that are not viable cannot invade. This proves the following theorem.

Theorem 3. Let $p$ be an evolving parameter, $V$ the viability region and $I^{*}(p)$ a branch of stable nontrivial equilibria corresponding to $p$. If $\lim _{p \rightarrow \partial V} I^{*}(p)=0$, then $\partial V$ is evolutionarily repelling.

Theorem 3 says that if the immigration rate goes continuously to zero when the viability boundary is approached, then this boundary is evolutionarily repelling; thus selection will move the dispersal strategy into the interior of the viability region.

\subsection{First example: Constant catastrophe rate}

We now study an example, in which the metapopulation exhibits a transcritical bifurcation, i.e., a continuous transition to extinction. We assume that neither the catastrophe rate nor the dispersal rate depend on local population size; the local growth rate is chosen as being logistic, $g(x)=a(1-x / K)$. 
(a)

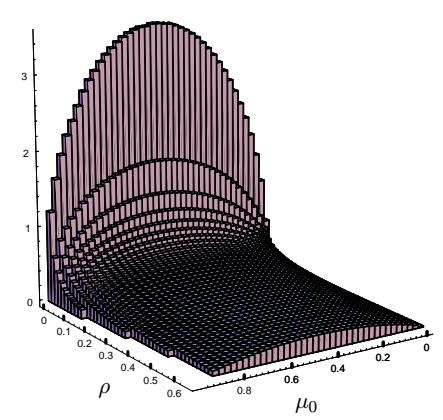

(b)

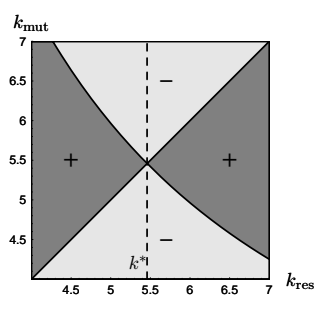

Figure 3: (a) Dependance of CSS dispersal rate $k^{*}$ on catastrophe rate $\mu_{0}$ and dispersal risk $\rho$ for the first example. Parameters: $a=1, K=1, \alpha=0.5$. (b) Pairwise invasibility plot for $\mu_{0}=0.8$ and $\rho=0.005$. The CSS dispersal rate is located at $k^{*} \approx 5.5$.

In this case the basic reproduction ratio is given by

$$
R^{\mathrm{res}}(I)=\pi \int_{0}^{1} \frac{2 k\left[1-y^{\frac{S(I)}{\mu}}\right]}{S(I)+k-a+y^{\frac{S(I)}{\mu}}[S(I)-k+a]} d y,
$$

with $S(I)=\sqrt{(k-a)^{2}+4 a I / K}$. Tedious but straightforward calculations show that $\frac{d}{d I} R^{\text {res }}(I) \leqslant 0$, so the function $R^{\text {res }}(I)$ is monotonically nonincreasing. Therefore, if $R_{0}^{\text {res }}>$ 1 , there exists a unique positive equilibrium, which is stable. If $R_{0}^{\text {res }}<1$, the extinct state is the only equilibrium. At the boundary of viability $R_{0}^{\text {res }}=1$ and the immigration rate goes continuously to zero. We thus have a transcritical bifurcation at $k=(a-\mu) / \rho$ and at $k=0$. See Fig. 1a for an example. According to Theorem 3 the boundaries are evolutionarily repelling.

In Fig. 3a there is always a continuously stable strategy in the viability region $0 \leqslant$ $\mu<1$. As studied by Parvinen et al. (2000), the dispersal rate exhibits an intermediate maximum with respect to catastrophe rate $\mu$. Increasing dispersal risk $\rho$ decreases the dispersal rate.

\section{Discontinuous transition to extinction}

In this section we study the case of discontinuous transition to extinction: here the equilibrium immigration rate first changes continuously, but then suddenly drops to zero as the dispersal rate $k$ varies. As a typical example we mention the case in which the equilibrium corresponding to $I^{*}=0$ loses its stability in a subcritical bifurcation (see Fig. $2 \mathrm{~b}$ ).

\subsection{A discontinuous transition to extinction is necessary for evolution- ary suicide}

Evolutionary suicide is an evolutionary process, during which an initially viable metapopulation adapts in such a way that it can no longer persist, see Ferrière (1999, 2000,in prep.). See also Matsuda and Abrams (1994).

Theorem 3 shows that, in the case of a continuous transition to extinction, the boundaries of viability are evolutionarily repelling. Therefore, a discontinuous transition to extinction is a necessary condition for evolutionary suicide. It is, however, not a sufficient condition, as we will see from our second and third example below. In the following section 


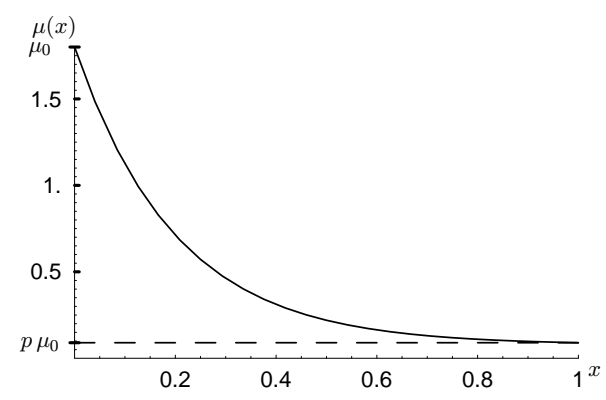

Figure 4: The catastrophe function $\mu(x)$ as described by (36) for $\mu_{0}=1.8, p=0.05$, $K=1, z=5$.

(a) $\rho=0.05$

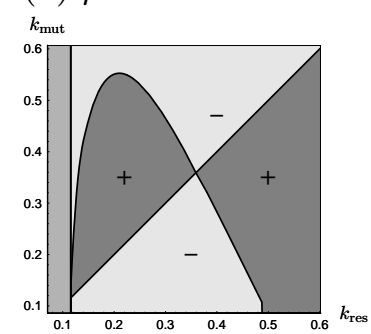

(b) $\rho=0.11$

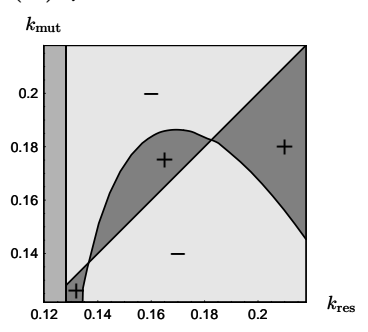

(c) $\rho=0.1175$

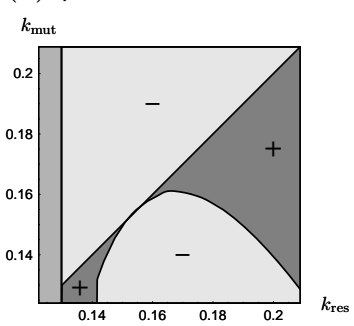

(d) $\rho=0.12$

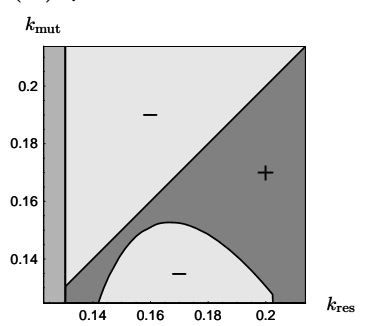

Figure 5: Pairwise invasibility plots illustrating the route to evolutionary suicide. The medium gray rectangle on the left side of each panel corresponds to values of the resident strategy $k$ for which the metapopulation is not viable. Local growth is logistic with $g(x)=a(1-x / K)$. Parameters: $\alpha=0.5, a=1, K=1$. The catastrophe function $\mu(x)$ is given by (36) with $\mu_{0}=1.8, p=0.05, z=5$.

we analyze how the phenomenon of evolutionary suicide can occur in the metapopulation model studied in this paper.

\subsection{Second example: Size-dependent catastrophe rate}

If the catastrophe rate is a decreasing function of the local population size and $R_{0}^{\text {res }}<1$, we can observe a special type of evolutionary bifurcation when increasing the dispersal risk $\rho$. In the following examples we use the catastrophe function

$$
\mu(x)=\mu_{0}\left[1-(1-p) \frac{1-\exp (-z x / K)}{1-\exp (-z)}\right] .
$$

This specific choice is used for illustration only. See Fig. 4 for an example. The number $\mu(0)=\mu_{0}$ describes the level of the catastrophe functions, it does not change its shape. The number $K$ is chosen to be the carrying capacity of the local growth function $g(x)$ and $p$ is the fraction of the catastrophe rate at the carrying capacity $K$ relative to $\mu_{0}$, thus $\mu(K)=p \mu_{0}$. The parameter $z$ describes the steepness of decrease.

With low dispersal risk $\rho$, there exists one CSS (Fig. 5a). When the risk increases, the viable region decreases and the CSS dispersal rate approaches the lower boundary of viability. When the risk is high enough, there appears another evolutionarily stable strategy that, however, is not convergence stable, i.e., it is an evolutionary repellor (Fig. 5b). 
(a) Evolutionary outcome

(b) Bifurcation with respect to $\rho$
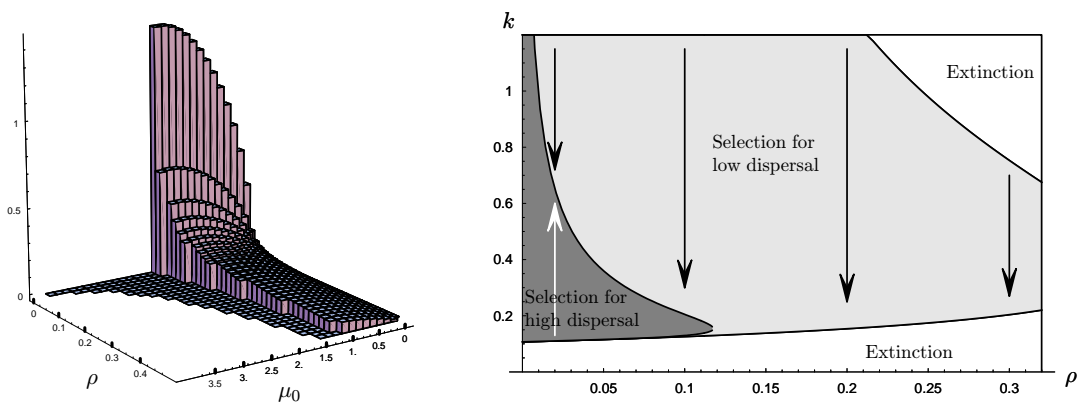

Figure 6: (a) Dependence of CSS dispersal rate $k^{*}$ on catastrophe rate $\mu_{0}$ and dispersal risk $\rho$ for the second example. For small dispersal risk and catastrophe rate there exists a CSS dispersal rate. When either dispersal risk or catastrophe rate is increased enough, an evolutionary bifurcation to evolutionary suicide occurs (gray part of bottom plane). The white part of the bottom plane corresponds to parameter combinations for which the metapopulation is not viable. (b) Bifurcation diagram with respect to $\rho$ for a cross-section at $\mu_{0}=1.8$. Dispersal rates at which selection favors lower dispersal rates are plotted in light gray; selection for higher dispersal rates is indicated by dark gray. In the white area the metapopulation is not viable. For low dispersal risk, a CSS dispersal rate exists. When the dispersal risk is increased, the domain in which selection favors higher dispersal rates shrinks and finally disappears. After that point, selection for lower dispersal rates leads to evolutionary suicide. Local growth is logistic, $g(x)=a(1-x / K)$. The catastrophe function is given by (36) with $z=5, p=0.05$. Other parameters: $\alpha=0.5, a=1, K=1$.

When the dispersal risk increases even more, these two singular strategies collide (Fig. 5c) and thereby disappear (Fig. 5d).

Once the regime depicted in Fig. $5 \mathrm{~d}$ has been reached, residents with a viable dispersal rate can always be invaded by some mutants with a lower dispersal rate. The dispersal rate will therefore decrease and will move closer and closer towards the lower boundary of viability. Once a resident at the lower boundary has become established, again a mutant with even lower dispersal rate can increase in population size. The mutant is not viable alone and therefore it cannot replace the resident. It will, however, move the resident population size away from its original attractor into the basin of attraction of the equilibrium that corresponds to extinction. Thus, the metapopulation will go extinct.

Fig. 6a shows the interplay of dispersal risk $\rho$ and catastrophe rate $\mu_{0}$ in setting the stage for evolutionary suicide. For low dispersal risks and catastrophe rates the viability boundaries are repelling and there is a continuously stable dispersal rate. When either dispersal risk or catastrophe rate is increased enough an evolutionary bifurcation to evolutionary suicide occurs as described above. Beyond that point, the viability region shrinks and finally disappears, leading to extinction of the metapopulation. Fig. $6 \mathrm{~b}$ illustrates this bifurcation structure along a cross-section at $\mu_{0}=1.8$.

\subsection{Third example: Allee effect}

Another ecological scenario for which we would expect a discontinuous transition to extinction arises from local population growth functions with an Allee effect, i.e., with a negative per capita growth rate at low population sizes (Allee et al. 1949).

The resulting bifurcation structure is similar to that in the second example: interme- 
(a) Growth functions

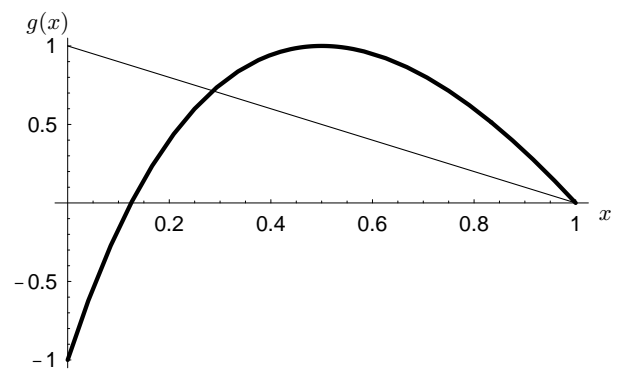

(b) Equilibrium values of $I$

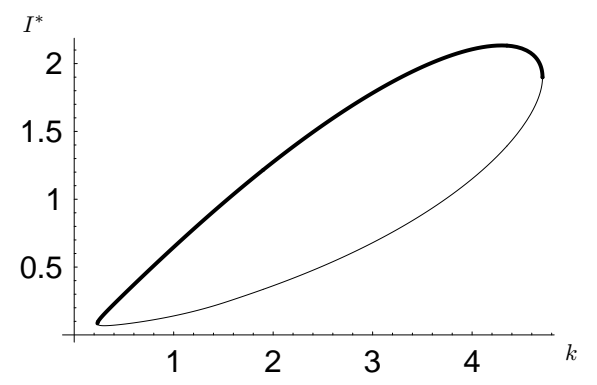

Figure 7: (a) Logistic growth function $g(x)=1-x$ (thin curve) and a growth function with Allee effect (thick curve) $g(x)=a \frac{x}{1+x}-b-c x$ with $a=18, b=1, c=8$. (b) Equilibrium values for the immigration rate $I$ in the case of an Allee effect. Stable equilibria lie on the thick curve, unstable ones on the thin curve. Parameters: $\alpha=0.5, \mu=0.2, \nu=0.1$.

diate dispersal risks and catastrophe rates result in a CSS dispersal rate. When either dispersal risk or catastrophe rate is increased enough, a bifurcation to evolutionary suicide occurs. See Fig. 8.

For low catastrophe rates, however, the situation is different. Selection towards low dispersal then leads to evolutionary suicide. This is remarkable: one would naively expect that only too high catastrophe rates may cause a metapopulation to go extinct. Yet in this example extinction also happens if there are too few catastrophes.

Is this finding compatible with Theorem 2, which states that without catastrophes the strategy not to disperse is a CSS? It is indeed compatible, as the remainder of this paragraph explains. When there are no catastrophes, all patches will reach the maximum population size $\tilde{x}$, therefore the average population size is $\tilde{x}$. Selection will favor low dispersal: after adaptation is completed, we have $k=0$. Therefore $\tilde{x}$ equals the carrying capacity of the growth function. Patches with very small population size would become empty if an Allee effect were present, but this is not a problem to the metapopulation since all local populations are large and no catastrophes occur. However, as soon as we introduce a little amount of catastrophes, the situation changes drastically. Catastrophes are so infrequent that low dispersal is still favored by selection. Yet, when dispersal becomes too low, a patch cannot be rescued after its population has been eradicated by a catastrophe. Since all patches will eventually experience a catastrophe, the metapopulation goes extinct.

Fig. 9 illustrates this situation. In the absence of catastrophes, the metapopulation is viable for any dispersal rate that is not too large. With positive catastrophe rate, the range of viable dispersal rates shrinks at both ends. In particular, the metapopulation is not viable for sufficiently small dispersal rates and a discontinuous transition to extinction arises that allows for evolutionary suicide.

\subsection{A discontinuous transition to extinction is not sufficient for evolu- tionary suicide}

According to Theorem 3, no evolutionary suicide can occur when the transition to extinction is continuous. As stated in Section 5.1, a discontinuous transition to extinction is therefore a necessary condition for evolutionary suicide. Nevertheless, the boundaries of the viability region can be repelling also in this case. Both our second and third example allow for some parameter values that bring about a discontinuous transition to extinction 
(a) Evolutionary outcome

(b) Bifurcation with respect to $\mu$
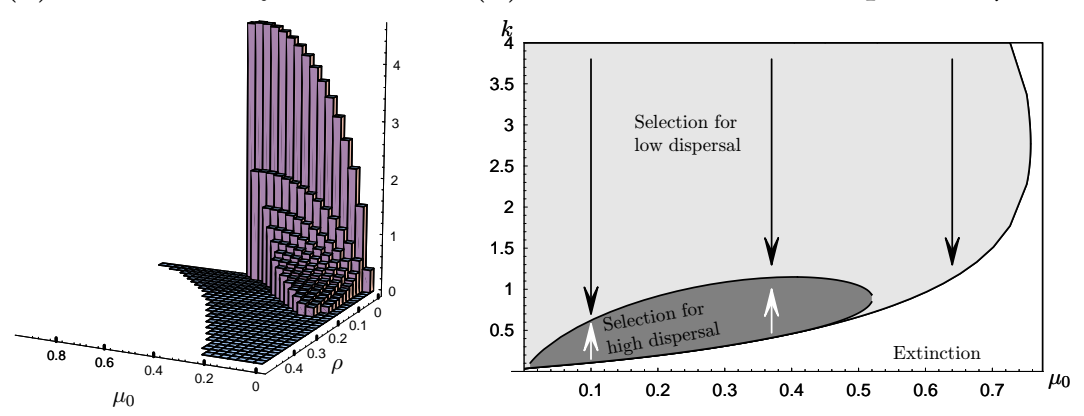

Figure 8: (a) Dependence of CSS dispersal rate $k^{*}$ on catastrophe rate $\mu_{0}$ and dispersal risk $\rho$ for the third example. For high dispersal risk and catastrophe rate we observe the same pattern as in Fig. 6: the range for which selection favors higher dispersal rates decreases and eventually disappears, leading to evolutionary suicide. For low catastrophe rates the situation is completely different: evolutionary suicide can result from an improvement of environmental conditions by decreasing $\mu_{0}$. (b) Bifurcation diagram with respect to $\mu_{0}$ for a cross-section at $\rho=0.05$. The region of parameter values where lower dispersal is selected for is plotted in light gray, and selection for higher dispersal occurs for the region plotted in dark gray. In the white area the metapopulation is not viable. Local growth exhibits an Allee effect according to Fig. 7a. Parameters: $\alpha=0.5, \mu(x)=\mu_{0}$.

without resulting in evolutionary suicide.

In the second example the catastrophe rate $\mu(x)$ is a decreasing function of the local population size $x$. If $\mu(0)>g(0)$, the basic reproduction ratio $R_{0}^{\text {res }}$ is negative for any dispersal rate. Therefore, either a discontinuous transition to extinction occurs (as in Fig. 1c) or the metapopulation is not viable for any dispersal rate $k$. When the dispersal risk $\rho$ is low enough, a CSS dispersal rate exists and no evolutionary suicide occurs (see Figs. 5a and 6).

In the third example we have $g(0)<0$ and the basic reproduction ratio $R_{0}^{\text {res }}$ is negative for any catastrophe rate $\mu$ and dispersal rate $k$. Like in the second example, there is either a discontinuous transition to extinction (Fig. 7b) or certain extinction. Also in this example there are parameter combinations of $\mu$ and $\rho$ that allow for a CSS dispersal rate (see Fig. 8).

\section{Discussion}

In this paper we have studied the evolution of dispersal and the potential for evolutionary suicide in a structured metapopulation model. We have proved that, in the absence of local catastrophes, selection will favor decreased dispersal rates: the strategy not to disperse is an ESS. In the presence of catastrophes, we have studied bifurcations that result from a parameter value crossing the boundary between regions of viability and extinction. If a continuous transition to extinction takes place (i.e. the immigration rate goes continuously to zero) when dispersal is varied, the extinction boundary is evolutionarily repelling and no evolutionary suicide can occur. We proved that a discontinuous transition to extinction (a positive immigration rate goes abruptly to zero at some parameter value) is a necessary but not a sufficient condition for evolutionary suicide. These findings are summarized in Fig. 10.

Two examples have illustrated that evolution does not necessarily optimize at the 


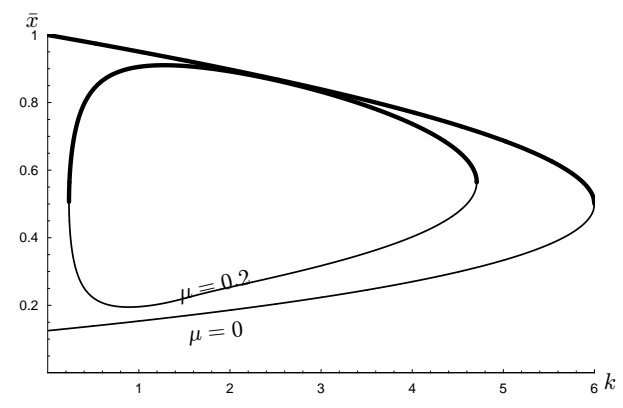

Figure 9: Average population size $\bar{x}$ at equilibrium for $\mu=0$ and $\mu=0.2$ when the growth function exhibits an Allee effect. Population sizes corresponding to stable equilibria lie on the thick curve, unstable ones on the thin curve. Parameters: $\alpha=0.5, \nu=0.1$.

\begin{tabular}{|c|c|c|c|}
\hline \multicolumn{2}{|c|}{ Without catastrophes } & $\longrightarrow$ & $\begin{array}{l}\text { Zero dispersal is evolutionarily } \\
\text { stable and convergence stable* }\end{array}$ \\
\hline \multirow[t]{2}{*}{$\frac{\mathscr{Q}}{\mathbb{d}}$} & $\begin{array}{l}\text { Continuous transition } \\
\text { to extinction }\end{array}$ & $\longrightarrow$ & $\begin{array}{l}\text { Usually intermediate dispersal } \\
\text { is evolutionarily stable and } \\
\text { convergence stable; no } \\
\text { evolutionary suicide }\end{array}$ \\
\hline & $\begin{array}{l}\text { Discontinuous transition } \\
\text { to extinction }\end{array}$ & & Evolutionary suicide \\
\hline
\end{tabular}

Figure 10: Summary of the different cases investigated in this paper. *: convergence stability applies if dispersal risk is positive.

population or metapopulation level (Metz et al. 996b). What is good for an individual is not always good for the metapopulation. Metapopulation extinction due to selfish individual adaptation is an extreme example of this general feature of frequency-dependent selection. Other real-life examples of this phenomenon are related to the tragedy of the commons (Hardin 1968).

To our knowledge, Matsuda and Abrams (1994) were the first to show in a mathematical model that frequency-dependent selection can lead to evolutionary suicide, or, as they called it, runaway evolution to self-extinction. They studied a population under asymmetric competition, in which, for some conditions, individuals of larger body size are always favoured by selection, leading to a continuous decrease in carrying capacity. Consequently, no evolutionary suicide is possible in the deterministic version of that model and Matsuda and Abrams correctly emphasize that, if the carrying capacity would reach zero at a finite body size, self-extinction could not occur. In this sense, our model and the one by Ferrière (2000) may be the first to demonstrate the existence of an evolutionarily attracting extinction boundary.

Yet, the study by Matsuda and Abrams (1994) makes another important point: whenever adaptation takes a biological population into or through regions of trait space that imply small carrying capacities, the evolving population can go extinct. Evidently, evolutionary suicide therefore occurs more easily under stochastic population dynamics than under deterministic ones. While conditions for the deterministic form of evolutionary suicide have been analyzed in this paper, we do not expect that similarly systematic conditions exist for the form of evolutionary suicide that requires populations of finite size.

Another repercussion of frequency-dependent selection in our model is even more strik- 
ing. In general, we are used to the phenomenon that, if the living conditions of a metapopulation become too unfavorable, the metapopulation will go extinct. This can result from increasing either the catastrophe rate or the dispersal risk in our model. However in the presence of an Allee effect and for low catastrophe rates, individuals select to disperse too little to re-colonize the patches after catastrophes have occurred. Under such conditions, making the environment more favorable will have a very strong negative effect on metapopulation viability: improving environmental conditions can sometimes send a population to extinction. 


\section{A Cumulative formulation of the model}

In this appendix we derive the expressions for the metapopulation steady states in the cases $\mu=0$ and $I=0$, which could not be dealt with using the shorthand notation (5)-(7) of the model. The nonlinear theory of general structured population models was presented by Diekmann et al. (2001) and adapted to structured metapopulation models by Gyllenberg et al. (1997). The specific model treated in this paper was formulated by Gyllenberg and Metz (1999).

Because we are only dealing with steady states we can replace equation (7) by its quasi-steady approximation

$$
I(t)=\pi \int_{0}^{\infty} k(x) x n(t, d x) .
$$

This approximation leads, of course, to precisely the same metapopulation steady states as the full model incorporating a disperser pool. In (37) the metapopulation state $n(t, \cdot)$ at time $t$ is a measure. If $n(t, \cdot)$ has a density, as in Section 2, we write (abusing notation) $n(t, x) d x$ instead of $n(t, d x)$.

Local population growth is modelled as a Markov process with local extinction as an absorbing state by prescribing the transition probabilities $u_{I}(t, x)$, that is, $u_{I}(t, x)(\omega)$ is the probability that a local population which had size $x$ at time 0 is still extant at time $t$ and has size in the set $\omega$. Reproduction (the formation of new local populations) is modelled by prescribing the reproduction kernel $\Lambda: \Lambda_{I}(t, x)(\omega)$ is the expected number of new local populations with "size-at-birth" in the set $\omega$, produced in the time interval $[0, t)$ by a local population which had size $x$ at time 0 . Our notation differs slightly from the one used by Diekmann et al. (2001).

A local population, which at time 0 had size $x$, will at time $t$ have size $X_{I}(t, x)$, provided it has not been wiped out by a local catastrophe. The probability that the population is still extant at time $t$ is

$$
\mathcal{F}_{I}(t, x)=\exp \left[-\int_{0}^{t} \mu\left(X_{I}(s, x)\right) d s\right] .
$$

Note that if $\mu=0$ (there are no catastrophes), $\mathcal{F}_{I} \equiv 1$. The transition probability of the local population size is given by the measure

$$
u_{I}(t, x)=\mathcal{F}_{I}(t, x) \delta_{X_{I}(t, x)} .
$$

When a local population is destroyed by a catastrophe, the patch it inhabited will immediately be recolonized by migrants arriving from other patches provided $I>0$. We consider this event as the simultaneous death of the local population and the birth of a new local population with size 0 . If $I=0$, there are no dispersers and there will still be death but no birth of a new population. The expected number of new local populations produced in the time interval $[0, t)$ by a local population having size $x$ at time 0 and subject to the input $I$ is therefore

$$
1-\mathcal{F}_{I}(t, x)
$$

and the reproduction kernel is

$$
\Lambda_{I}(t, x)= \begin{cases}\left(1-\mathcal{F}_{I}(t, x)\right) \delta_{0} & \text { if } I>0 \\ 0 & \text { if } I=0 .\end{cases}
$$


Let us first consider the case $\mu=0$. Then there are neither births nor deaths of local populations, that is, $\Lambda_{I} \equiv 0$. The system is therefore at equilibrium if and only if the local dynamics given by $u_{I}$ is at equilibrium:

$$
n=\int u_{I}(t, x) n(d x)
$$

for all $t$. Inserting (39) (with $\mathcal{F}_{I} \equiv 1$ ) into (42) one gets

$$
n=\int \delta_{X_{I}(t, x)} n(d x), \quad t \geqslant 0
$$

from which it follows that $n=\delta_{\tilde{x}(I)}$. We have now shown that $\delta_{\tilde{x}(I)}$ is the steady state, where $I$ is solved from

$$
I=\pi \int k(x) x \delta_{\tilde{x}(I)}(d x)
$$

that is, from

$$
I=\pi k(\tilde{x}(I)) \tilde{x}(I)
$$

Let us now turn to the case $I=0$. In this case we still have $\Lambda_{I} \equiv 0$. Because $f(0,0)=0$, we have $X_{I}(t, 0)=0$ for all $t$. Therefore $u_{I}(t, 0)=\delta_{0}$ for $I=0$ and consequently (42) can be satisfied by $n=\delta_{0}$ only. Thus $I=0$ implies $n=\delta_{0}$. 


\section{References}

Allee, W. C., A. Emerson, T. Park, and K. Schmidt (1949). Principles of Animal Ecology. Saunders, Philadelphia.

Christiansen, F. B. (1991). On conditions for evolutionary stability for a continuously varying character. Am. Nat. 138, 37-50.

Dieckmann, U. (1997). Can adaptive dynamics invade? Trends Ecol. Evol. 12, 128-131.

Diekmann, O., M. Gyllenberg, H. Huang, M. Kirkilionis, J. A. J. Metz, and H. R. Thieme (to appear in 2001). On the formulation and analysis of general deterministic structured population models. II. Nonlinear theory. J. Math Biol..

Diekmann, O., M. Gyllenberg, J. A. J. Metz, and H. R. Thieme (1998). On the formulation and analysis of general deterministic structured population models. I. Linear theory. J. Math. Biol. 36, $349-388$.

Diekmann, O., M. Gyllenberg, and H. R. Thieme (2000). Lack of uniqueness in transport equations with a nonlocal nonlinearity. Mathematical Models and Methods in Applied Sciences 10, 581-592.

Eshel, I. (1983). Evolutionary and continuous stability. J. Theor. Biol. 103, 99-111.

Ferrière, R. (1999). Adaptive response of dispersal to habitat loss: evolutionary rescue, traps and suicide. In Spatial Ecology Conference on Habitat Loss: Ecological, Evolutionary and Genetic Consequences. Helsinki, Finland, 7-12. September 1999. http://www.helsinki.fi/ml/ekol/se/hleegc1.html.

Ferrière, R. (2000). Adaptive responses to environmental threats: evolutionary suicide, insurance, and rescue. Options Spring 2000, 12-16.

Ferrière, R. and C. Cadet (in prep.). Adaptive responses in metapopulations subject to environmental change: evolutionary rescue and evolutionary suicide.

Gandon, S. (1999). Kin competition, the cost of inbreeding and the evolution of dispersal. J. Theor. Biol. 200, 245-364.

Gandon, S. and Y. Michalakis (1999). Evolutionarily stable dispersal rate in a metapopulation with extinctions and kin competition. J. Theor. Biol. 199, 275-290.

Geritz, S. A. H., É. Kisdi, G. Meszéna, and J. A. J. Metz (1998). Evolutionarily singular strategies and the adaptive growth and branching of the evolutionary tree. Evol. Ecol. 12, 35-57.

Geritz, S. A. H., J. A. J. Metz, É. Kisdi, and G. Meszéna (1997). Dynamics of adaptation and evolutionary branching. Phys. Rev. Lett. 78, 2024-2027.

Gyllenberg, M. and I. A. Hanski (1992). Single-species metapopulation dynamics: a structured model. Theor. Popul. Biol. 42, 35-62.

Gyllenberg, M. and I. A. Hanski (1997). Habitat deterioration, habitat destruction and metapopulation persistence in a heterogeneous landscape. Theor. Popul. Biol. 52, 198215.

Gyllenberg, M., I. A. Hanski, and A. Hastings (1997). Structured metapopulation models. In I. A. Hanski and M. E. Gilpin (Eds.), Metapopulation Biology: Ecology, Genetics, and Evolution, pp. 93-122. Academic Press.

Gyllenberg, M. and J. A. J. Metz (1999). On fitness in structured metapopulations. Interim report IR-99-037, IIASA, Laxenburg, Austria. http://www.iiasa.ac.at/cgibin/pubsrch?IR99037. 
Hanski, I. A. and M. Gyllenberg (1993). Two general metapopulation models and the core-satellite species hypothesis. Am. Nat. 142, 17-41.

Hanski, I. A. and M. Gyllenberg (1997). Uniting two general patterns in the distribution of species. Science 275, 397-400.

Hardin, G. (1968). The tragedy of the commons. Science 162, 1243-1248.

Hastings, A. (1983). Can spatial variation alone lead to selection for dispersal. Theor. Pop. biol. 24, 244-251.

Heino, M. and I. Hanski (2000). Evolution of migration rate in a spatially realistic metapopulation model. Interim report IR-00-044, IIASA, Laxenburg, Austria. http://www.iiasa.ac.at/cgi-bin/pubsrch?IR00044.

Levins, R. (1969). Some demographic and genetic consequenses of environmental heterogeneity for biological control. Bill. Entomol. Soc. Am. 15, 237-240.

Levins, R. (1970). Extinction. In M. Gerstenhaber (Ed.), Some Mathematical Problems in Biology, pp. 77-107. American Mathematical Society, Providence, RI.

Matsuda, H. and P. A. Abrams (1994). Runaway evolution to self-extinction under asymmetrical competition. Evolution 48, 1764-1772.

Maynard Smith, J. (1982). Evolution and the theory of games. Cambridge University Press, Cambridge.

Maynard Smith, J. and G. R. Price (1973). The logic of animal conflict. Nature 246, 15-18.

Metz, J. A. J., S. A. H. Geritz, G. Meszéna, F. J. A. Jacobs, and J. S. van Heerwaarden (1996a). Adaptive dynamics, a geometrical study of the consequenses of nearly faithful reproduction. In S. J. van Strien and S. M. Verduyn Lunel (Eds.), Stochastic and Spatial Structures of Dynamical Systems, pp. 183-231. North-Holland,Amsterdam.

Metz, J. A. J. and M. Gyllenberg (to appear in 2001). How should we define fitness in structured metapopulation models? Including an application to the calculation of ES dispersal strategies. Proc. Royal Soc. B.

Metz, J. A. J., S. D. Mylius, and O. Diekmann (1996b). When does evolution optimize? On the relation between types of density dependence and evolutionarily stable life-history parameters. Working paper WP-96-004, IIASA, Laxenburg, Austria. http://www.iiasa.ac.at/cgi-bin/pubsrch?WP96004.

Parvinen, K. (1999). Evolution of migration in a metapopulation. Bull. Math. Biol. 61, $531-550$.

Parvinen, K., U. Dieckmann, M. Gyllenberg, and J. A. J. Metz (2000). Evolution of dispersal in metapopulations with local density dependence and demographic stochasticity. Interim report IR-00-035, IIASA, Laxenburg, Austria. http://www.iiasa.ac.at/cgibin/pubsrch?IR00035.

Ronce, O., F. Perret, and I. Olivieri (2000). Evolutionary stable dispersal rates do not always increase with local extinction rates. Am. Nat. 155, 485-496.

Taylor, P. D. (1989). Evolutionary stability in one-parameter models under weak selection. Theor. Popul. Biol. 36, 125-143. 